\title{
LAS PRIMERAS CONQUISTAS FEMENINAS: ESCRITURA, PRENSA Y EDUCACIÓN (1830-1916)
}

\author{
THE FIRST FEMALE CONQUESTS: \\ WRITING, PRESS AND EDUCATION \\ (1830-1916)
}

\author{
Lucrecia Infante Vargas \\ (ID) orcid.org/0000-0002-6779-711X \\ Universidad Nacional Autónoma de México \\ México \\ lucrecia_infante@outlook.com
}

\begin{abstract}
Reading and writing are vital tools for inhabiting and transcending the world. The appropriation that women made of reading and writing was a fundamental precursor for the reception of the first ideas that, in favor of their emancipation, fought for their access to higher education during the 19th century. And, in the case of our country, for the first civil feminist demands to emerge at the dawn of the twentieth century. The present text gives an account of this process and its impact on the gestation of organized Mexican women's action.
\end{abstract}

Keywords: education, periodical press, women authors.

\begin{abstract}
Resumen
Leer y escribir son herramientas vitales para habitar y trascender el mundo. La apropiación que las mujeres hicieron de la lectura y la escritura fue un antecedente fundamental para la recepción de las primeras ideas que, a favor de su emancipación, pugnaron por su acceso a la educación superior durante el siglo XIX. Y, en el caso de nuestro país, para que surgieran las primeras demandas civiles feministas en los albores del siglo XX. El presente texto da cuenta de este proceso y de su impronta en la gestación de la acción organizada de las mujeres mexicanas.
\end{abstract}

Palabras clave: educación, escritoras, prensa. 


\section{Algunos antecedentes}

Los vestigios de la escritura de las mujeres en nuestro país pueden documentarse al menos desde el siglo XVII, pero no sólo en la figura de la excepcionalmente conocida monja jerónima Juana Inés de Asbaje y Ramírez de Santillana (1651-1695), Ilamada la Décima musa y Fénix de México, Juana Inés de la Cruz, sino también en la vasta obra producida precisamente, al interior de los conventos, por muchas de las religiosas que, además de reproducir libros -es decir, copiarlos a mano-, escribían sus obligados ejercicios espirituales, poesía devota, las crónicas de fundación de sus órdenes e incluso la biografía de otras monjas, beatas y colegialas ejemplares. ${ }^{1}$

De acuerdo con la historiadora Josefina Muriel, una de las pioneras en el estudio de las órdenes religiosas femeninas novohispanas, entre 1557 y 1790 este conjunto de escritos pudo llegar a sumar más de un centenar (Muriel, 1946), aunque esto es difícil de precisar, pues en su mayoría fueron desconocidos, ya que por lo general pasaban a manos de algún fraile que tras corregir las fallas de estilo, precisión e incluso caligrafía-que se presumían inherentes a la redacción femenina-, los firmaba con su nombre. Todos estos textos tampoco fueron reconocidos como productos intelectuales (al menos no en aquella primera versión escrita por la religiosa), debido a la idea de que dicha escritura no representaba un acto creativo en sí, puesto que por un lado respondía tan sólo al cumplimiento de instrucciones ajenas (la del preceptor, el confesor o cualquier otra autoridad provincial que supervisaba la vida al interior de los conventos); por otro, a que presumiblemente no hacía más que reproducir (copiar, imitar), el canon estético o conceptual establecido para dichos géneros narrativos $y$, en dicho sentido, no merecía ser considerado un resul-

\footnotetext{
1 Para una exposición más amplia de varios de los espacios de educación informal y las prácticas de escritura que se mencionan en el presente texto, véanse dos investigaciones anteriores de las cuales retomo una parte sustancial de los planteamientos aquí expuestos (Infante Vargas, 2014 y 2017).
}

tado original del pensamiento, o propio de una labor intelectual.

Lejos de ello, y gracias a las crecientes investigaciones generadas al respecto, hoy es sabido que las fundaciones religiosas (más de veinte hacia el siglo XVII, tanto en la Ciudad de México como en Guadalajara, Puebla, San Luis Potosí, Querétaro, Oaxaca y Michoacán) sí constituyeron un espacio de educación y formación intelectual para las mujeres -niñas, jóvenes solteras y mujeres adultas de diversos grupos étnicos y diferentes estratos sociales- que tuvieron la oportunidad de ingresar a ellas (Muriel, 2000).

Otras muchas historias de mujeres que al ingresar al convento -o a un colegio anexo al mismo- encontraron una puerta de acceso a la educación, y con ello de transformación en sus vidas, son también un ejemplo del papel que jugaron los conventos como instancias de acceso a la cultura y con ello a diversas posibilidades de relación con el mundo. Como mínima muestra, y sólo como ello, está el caso de sor Josefa de San Lorenzo, quien habiendo ingresado analfabeta al convento del mismo nombre, llegó a ser una importante y reconocida escritora de poesía devota.

Las escuelas de enseñanza básica y las llamadas Amigas son otros ámbitos no considerados como un antecedente fundamental del acceso de las mujeres a la escritura. En el primer caso, las escuelas conocidas como de "primeras letras", de acuerdo con las instrucciones de la Cédula dictada por Felipe II en 1604, se implementaron en todo el orbe novohispano para que niños y niñas recibieran dicha educación elemental. En el segundo, la muy temprana figura de la Amiga (pequeñas escuelas instaladas en la propia casa de las mujeres -mínima, o medianamente instruidas, y por lo general viudas-); espacios intermedios entre los muros familiares y los de la instrucción formal en donde además del aprendizaje del catecismo y las entonces consideradas labores femeninas (costura, bordado y cocina, entre otras), se enseñaban también las primeras letras, dentro de lo cual solía incluirse el aprendizaje de la escritura. 
Al igual que en el caso de los conventos, la apreciación que por lo general se tiene de ambos espacios minimiza su labor como centros educativos bajo el supuesto de que, en estricto sentido, no se brindaba una instrucción formal. Por el contrario, la trascendencia y los impactos de su actividad se confirma, por ejemplo, con la apertura en 1755 de la primera Amiga pública y gratuita (inaugurada como anexo del prestigiado colegio de monjas La Enseñanza o Colegio del Pilar). Un proyecto de largo alcance que con el propósito expreso de brindar educación básica a las niñas huérfanas y de bajos recursos fue auspiciado por el Ayuntamiento de la Ciudad de México, que cumplió con este fin todavía hasta la segunda mitad del siglo XIX.

Pero no fue sólo en estos espacios donde, de manera formal (la Amiga y las escasas aulas escolares) o informal (en el ámbito familiar) ciertos sectores de mujeres aprendieron, generación tras generación, a leer y a escribir. Por el contrario, su entrenamiento en dichas herramientas de acceso al conocimiento se registra también en otro escenario crucial para el desarrollo de su vínculo con la palabra escrita: las tertulias.

Estos espacios de reunión y convivencia social, promovidos en Nueva España por lo menos desde 1760 (en casa, por ejemplo, de doña Lorenza Martín Romero), llegaron a convertirse en sitios predilectos para el intercambio literario e incluso la conspiración política, como es el caso de las reuniones presididas por doña Mariana Rodríguez del Toro, o por la famosa María Ignacia Rodríguez -mejor conocida como La Güera Rodríguez-, e incluso por las también conocidas Leona Vicario y Josefa Ortiz de Domínguez.

Así pues, y a semejanza de los prestigiados salones en Francia, las tertulias representaron una vía de acceso -indirecto pero constante- al conocimiento de los autores y temas de discusión vigentes en la ciencia, la filosofía o la política, así como una oportunidad para insertarse en el mundo de la escritura. En otras palabras, estos espacios fueron aprovechados por las mujeres para desarrollar habilidades vinculadas con el mundo de las letras, tales como la lectura -individual y en grupos-, la declamación o el intercambio de correspondencia personal y ensayos de orden literario.

Un caso aparte, y de gran interés pero lamentablemente aún sin estudios suficientes en nuestro país, es el de la escritura privada, esto es, aquella realizada desde una intención personal e íntima, como la redacción de una carta, un diario personal o de viaje.

La cercanía con este tipo de escritura que tuvieron ciertos grupos de mujeres en México se constata en los escasos pero generosos archivos epistolares que milagrosamente aún se conservan y cuya procedencia -en términos generales-corresponde a la pluma de mujeres pertenecientes a las familias de las élites social y cultural de los siglos XVIII y XIX.

Entre ellas destacan María Antonia Trebustos, María Josefa Rodríguez de Pedroso de la Cotera y Rivas Cacho, así como Josefa Villamil (primera, segunda y tercera condesas de Regla, respectivamente); María Ignacia Rodríguez de Velasco y Osorio (mejor conocida como La Güera Rodríguez y madre también de Josefa Villamil), Magdalena Catarina Dávalos y Orozco (Condesa de Miravalle), María Josefa Velasco y Obando, Antonia González Echeverría viuda de Agüero, Leona Vicario, Ana Iraeta de Mier, Mariana Rodríguez del Toro, María Velázquez de León, Concepción Lombardo de Miramón y los diarios de viaje de las hermanas Enriqueta y Ernestina Larrainzar, entre otras.

Otro referente olvidado es el Álbum, un pequeño cuaderno, casi del tamaño de un misal, en cuyas páginas las jóvenes (o sus admiradores y pretendientes) reproducen los poemas de sus autores favoritos, y por el cual, gracias a las muchas relecturas necesariamente requeridas para memorizar los versos transcritos, las mujeres se apropian no sólo del poema en cuestión, sino también de sus elementos formales: el lenguaje, el ritmo, la métrica.

En este sentido, la trascendencia de esta práctica de escritura es tal que, años más tarde, el Álbum daría nombre a todo un es- 
tilo de escritura en la prensa dirigida a las mujeres. Más aún, el éxito de la fórmula es tal, que uno de los impresores que mostraron gran interés por el mercado de revistas para mujeres, Juan R. Navarro, bautizaría con este nombre a uno de sus mayores éxitos editoriales: Álbum de las Señoritas. Revista de literatura y variedades, publicado por vez primera en 1856.

Asimismo, y como se mencionará más adelante, 27 años después, en 1883, éste será el nombre que retome uno de los impresos para mujeres de mayor importancia y duración en nuestro país, El Álbum de la mujer, dirigido durante casi una década por Concepción Gimeno de Flacquer, española avecindada en México.

\section{El arribo de las mujeres a la cultura impresa}

El breve recuento hasta aquí realizado de una escritura femenina no reconocida como tal, pero acontecida en la redacción de cartas, diarios o crónicas conventuales; la lectura individual -y en grupo- en las salas comunes de costura y bordado; la memorización y recitado de poemas en las tertulias y demás espacios de sociabilidad; el aprendizaje de un idioma diferente al propio mediante el ejercicio de la traducción; e inclusive la práctica de la tipografía y la encuadernación es, en suma, un antecedente contundente del proceso por el cual ciertos sectores de mujeres accedieron a la escritura, y que nos ayuda a explicar que ya desde 1805, en las páginas del primer impreso cotidiano de la Nueva España, el Diario de México, se publiquen remitidos y cartas firmados por puño y letra de una mujer.

Asimismo, a partir de entonces y hasta 1838, año en que surge la primera publicación periódica dirigida expresamente a un público femenino -el Calendario de las Señoritas Mexicanas, editado anualmente por Mariano Galván hasta 1843-, la presencia escrita de las mujeres en los medios impresos se mantuvo constante y progresiva, ya mediante el simple envío de saludos y felicitaciones al editor o bien en la forma de adivinanzas, charadas, poemas y solicitud de consejos.

Uno de los muchos textos del Diario de México da testimonio de cómo la escritura se convierte en un vehículo a través del cual las mujeres consiguen exponer sus ideas en el ámbito de lo público, tránsito sustancial en su futura configuración como ciudadanas. Se trata de la "Carta de una señorita a otra...", enviada por Pepita al Diario de México en 1806, quien deja entrever una ligera burla (¿o un refinado sentido del humor?) cuando se lamenta por no contar con la capacidad intelectual, la inteligencia necesaria para representar a sus congéneres:

A la verdad, querida amiga, si tuviera una bien cortada pluma, un estilo florido, y la más persuasiva elocuencia, tomaría con ardoroso interés la defensa de mi sexo, mas ¿̇qué puede hacer una mujer sin instrucción, que no tuvo otro patrimonio que la estupidez, que nació para vivir y morir sepultada en la ignorancia? (“Pepita”, 1810.)

De igual modo, la publicación en 1825 del Calendario dedicado a las señoritas Americanas, especialmente a las patriotas. Por el Pensador Mexicano, publicación elaborada por José Joaquín Fernández de Lizardi -conocido también en otro de sus seudónimos como El Periquillo Sarniento-, es una muestra más del posicionamiento de las mujeres como un público lector. Pero no sólo eso, este impreso, en estricto sentido el primero en México dirigido expresamente a las mujeres, justifica su aparición con el postulado de que la naciente patria debe un reconocimiento a todas las mujeres que protagonizaron acciones heroicas durante la guerra de independencia.

No resulta casual, creo, que además del acostumbrado santoral y de un conjunto de versos inspirados en la heroicidad antes mencionada, las figuras seleccionadas por el Calendario -María Leona Vicario, Mariana Rodríguez de Lazarín, Manuela Herrera y María Fermina Rivera-, también se vinculen por haber generado un registro escrito de su participación en la guerra de independencia. 
El caso de Leona Vicario es, quizá, el que mejor muestra la trascendencia cultural de lo antes dicho, pues además de su afición por la lectura y la traducción, protagonizó uno de los episodios que ilustra el papel de la palabra impresa como una expresión de la naciente modernidad política y, en dicho sentido, de la importancia que para las mujeres tuvo asimismo la escritura como una herramienta cultural que, gradualmente, les permitió posicionarse como sujetos públicos.

El acontecimiento referido ocurre en 1831, cuando el conocido y entonces Ministro de Relaciones, Lucas Alamán, publica un texto en el que acusa a Leona Vicario de aprovechar su vínculo matrimonial con el también conocido -y adversario político de AlamánAndrés Quintana Roo, para adjudicarse la posesión de una serie de fincas que, en calidad de pensión, el gobierno le otorgó en retribución a sus acciones en favor del movimiento insurgente; acciones que, a diferencia de la opinión emitida por Fernández de Lizardi, el señor Alamán no consideraba merecedoras de tal reconocimiento, puesto que su motivación fue en realidad "[...] cierto heroísmo romanesco [sic] que el que sepa algo del influjo de las pasiones, sobre todo en el bello sexo, aunque no haya leído a Madame de Staël, podrá atribuir a otro principio menos patriótico"(Alamán, 1831).

La respuesta de Leona, publicada 12 días después en el Federalista Mexicano, subrayó enfáticamente el error en que incurría el ministro Alamán, así como el agravio que le producía la suposición de que su apoyo al ejército insurgente fuera el resultado de sus afecciones sentimentales. Asimismo, y en con un simbólico empleo en femenino de la primera persona del plural, nosotras, afirmó:

No sólo el amor es el móvil de las acciones de las mujeres; que ellas son capaces de todos los entusiasmos, y que los deseos de gloria y de la libertad de la patria no les son unos sentimientos extraños [...] Si M. Staël atribuye algunas acciones de patriotismo en las mujeres a la pasión amorosa, esto no probará jamás que sean incapaces de ser patriotas, cuando el amor no las estimula a que lo sean. Por lo que a mí toca, sé decir que mis acciones y opiniones han sido siempre muy libres, nadie ha influido absolutamente en ellas, y en este punto he obrado siempre con total independencia [...] Me persuado que así serán todas las mujeres, exceptuando a las muy estúpidas, y a las que por efecto de su educación hayan contraído un hábito servil. De ambas clases también hay muchísimos hombres [...] Aseguro a Usted, Sr. Alamán, que me es sumamente sensible que un paisano mío, como es Usted, se empeñe en que aparezca manchada la reputación de una compatriota suya, que fue la única mexicana acomodada que tomó una parte activa en la emancipación de la patria. En todas las naciones del mundo, ha sido apreciado el patriotismo de las mujeres: ¿por qué, pues, mis paisanos, aunque no sean todos, han querido ridiculizarlo como si fuera un sentimiento impropio en ellas? ¿Qué tiene de extraño ni ridículo el que una mujer ame a su patria y le preste los servicios que pueda para que a éstos se les dé, por burla, el título de heroísmo romanesco? (Vicario, 1831).

El plural nosotras, empleada por Leona Vicario -nosotras las mujeres- permanece a partir de esta década en los diversos medios impresos atendidos por las lectoras. Una voz en plural que insiste, una y otra vez, en su interés, en su necesidad por adentrarse cada vez más en los terrenos de la palabra escrita. En dicho sentido, el surgimiento de la primera publicación periódica dedicada en exclusiva a las mujeres, el ya mencionado Calendario de las Señoritas Mexicanas, publicado en 1838 por uno de los editores más importantes de la época, Mariano Galván, es una expresión contundente del ingreso de las mujeres a los circuitos de la cultura impresa como agentes activos de dicho fenómeno, al consolidarse como un mercado lector específico.

A partir de entonces, y a lo largo de las dos décadas siguientes, más de una veintena de publicaciones femeninas aparecen, en un ritmo promedio de cinco a seis cada año. Lo único que por momentos interrumpe esa continuidad son los conflictos armados de orden mayor (como la guerra de 1847), que de 
forma directa rompen con el orden de lo cotidiano e impiden la llegada de los suministros básicos en las imprentas: la tinta y el papel.

Entre algunas de las más importantes se encuentran El Museo Yucateco (1841), que bajo la batuta de don Justo Sierra O’Reilly consigue aparecer cada mes, y durante un año, en la ciudad de Campeche; el Semanario de las Señoritas Mexicanas. Educación científica, moral y literaria del bello sexo (18411842), editado en la Ciudad de México por Isidro Rafael Gondra; El apuntador. Semanario de teatros, costumbres, literatura y variedades (1841), revista publicada por Ignacio Cumplido, también impresor del Presente Amistoso Dedicado a las Señoritas Mexicanas (una verdadera joya tipográfica), cuyos tomos anuales aparecen en 1847, 1851 y 1852.

De las publicaciones realizadas durante la década siguiente destacan La semana de las señoritas Mexicanas, que en sus distintas épocas (1850, 1851 y 1852) publicó la imprenta de Juan R. Navarro, también responsable de la publicación de otro par de importantes revistas, La Camelia. Semanario de literatura, variedades, teatros, modas. Dedicado a las señoritas mexicanas, que circuló de 1852 a 1853, y El Álbum de las Señoritas (1855-1856).

Este progresivo incremento de publicaciones femeninas es paralelo al aumento de las mujeres que comienzan a participar en ellas como colaboradoras. Así, mientras que en 1838 sólo se publica un texto firmado por una mujer con su nombre y apellido real, hacia 1859 el número de firmas se incrementa a 39. Si en términos cuantitativos el aumento es indiscutible, el terreno ganado por las escritoras es claro también en la ampliación de las temáticas que abordan y de los géneros literarios en que lo hacen. Con relación a este último aspecto, aun cuando la poesía se mantiene como género predilecto, aumenta muy significativamente la cantidad de traducciones, cuentos, novelas y ensayos. Cabe mencionar también que ya no sólo escriben sobre los tópicos románticos de costumbre (el amor maternal, la muerte, la naturaleza - las desavenencias amorosas); tampoco sobre los rubros que, para entonces, se han convertido en secciones indispensables de estas revistas: moda, consejos domésticos, - la crónica semanal de eventos sociales. Ahora escriben sobre asuntos que -así lo afirman- les atañen e inconforman; en primer lugar, el escaso nivel de instrucción escolar de las mujeres, así como ciertos comportamientos masculinos que consideran moralmente ofensivos (como el adulterio). De igual modo es relevante que, aunque todavía de manera esporádica, se publican traducciones de notas que informan sobre los avances del sufragismo en Inglaterra y los Estados Unidos de Norteamérica.

La sucesiva disminución del seudónimo es también un cambio de suma importancia a lo largo de estas décadas. Por lo general, ya sólo se recurre a esta estrategia cuando los temas atendidos en el poema o el ensayo pueden poner en riesgo la honorabilidad de las escritoras, como aquellos que aluden a la experiencia de la sensualidad; o bien cuando se ejerce una crítica todavía más aguda sobre alguno de los temas vinculados a la condición social de las mujeres ya antes referidos: la desigualdad salarial entre los sexos, la negativa a que las mujeres accedan a la educación superior y, de manera muy especial, el menosprecio a sus capacidades intelectuales.

Es un momento oportuno para señalar que el ingreso de las mujeres al mundo de la palabra impresa no fue una batalla fácil de librar, como quizá parezca por lo hasta aquí narrado. Por el contrario, sus protagonistas enfrentaron una gran cantidad de conflictos, derivados todos, en general, de dos imaginarios culturales de difícil transformación. El primero, la presunta incapacidad natural de las mujeres para ejercer cualquier actividad de orden intelectual; el segundo, las presumibles consecuencias negativas que el oficio de la escritura podía generar en el desempeño de las mujeres como madres y esposas. Lo cual, en términos generales, manifestaba el temor a que dichos roles fueran desatendidos o desvalorizados por aquéllas, lo que suponía un peligroso desorden social.

En este sentido, la incursión en un territorio predominantemente masculino, y su conquista, no estuvo exenta de víctimas. 
Las tempranas y cercanas muertes de Teresa Vera (1834-1859) y Dolores Guerrero (1833-1858) fueron una triste prueba de que no bastaba ser inteligente y talentosa para convertirse en una escritora aplaudida, pero sobre todo, aceptada por la sociedad.

Estas dos jóvenes gozaron de una educación privilegiada, es decir, por encima del nivel acostumbrado de la enseñanza básica brindada a las mujeres entonces, y su obra escrita (en especial la poética) fue incluso elogiada por los círculos literarios que frecuentaban, tanto en sus entidades de origen, Veracruz y Durango, como en la capital del país. Ambas se quitaron la vida a causa de una presunta decepción amorosa que, además, puso en duda su decencia y honorabilidad. Sobre todo en el caso de Teresa, pues el periódico al que había enviado un poema de amor prohibido (declaraba su apasionado afecto por un conocido profesor que era casado) reveló la identidad de la poetisa, que había sido encubierta por ella con un seudónimo.

Con todo, las mujeres perseveraron en su decisión y tejieron diversos mecanismos para sobrepasar la hostilidad, la burla e incluso el escándalo que entre algunos sectores generaba su manifiesto deseo de ingresar a la república de las letras. Uno de los más sobresalientes fue la creación de las llamadas Amistades Románticas, una suerte de red de intercambio epistolar entre las poetas y sus lectoras, que llegó a convertirse en todo un género literario, en especial de la poesía.

Otra importante estrategia de acompañamiento fue la creación de equipos editoriales conformados exclusivamente por mujeres; grupos de colaboración reunidos en torno de prestigiadas revistas literarias que, además de permanecer unidos durante dos o tres años (periodos de tiempo considerablemente largos, si se toma en cuenta la volatilidad de las publicaciones decimonónicas), incrementan sus filas, e incluso se mantienen unidos al trasladarse de un impreso a otro.

En el mismo sentido, algunas de las revistas literarias que abrieron las puertas a estos equipos de colaboración editorial fueron El Anáhuac, dirigida por Manuel Payno y ór- gano de difusión de la prestigiada Sociedad Netzahualcóyotl, y El Renacimiento, fundada y dirigida, entre otros, por los prestigiados escritores Ignacio Ramírez, Guillermo Prieto y Justo Sierra.

La demarcación de este nuevo continente letrado en el que las mujeres escribían de sí mismas a partir de su vivencia, de su experiencia particular, fue asimismo la consolidación de un tránsito crucial en esta historia de apropiación de la palabra escrita: el paso de lectoras a redactoras, y el cruce de la frontera privada hacia el territorio de lo público.

\section{Estudiar y escribir: las primeras conquistas}

El surgimiento de la primera revista para mujeres editada por ellas mismas es un parteaguas fundamental en esta historia. La publicación de La Siempreviva (1870-1872), dirigida y redactada en Mérida, Yucatán, por las también oriundas de dicha península Rita Cetina Gutiérrez, Cristina Farfán y Gertrudis Tenorio Zavala inaugura el último tránsito en el proceso de apropiación de las mujeres de la escritura y, al mismo tiempo, da cuenta de por qué la demanda del derecho de las mujeres a la educación superior fue una exigencia permanente en las páginas de todas la revistas femeninas que conforman esta etapa final del siglo XIX y los primeros años del XX.

\section{Figura 1 Portada de La Siempreviva}

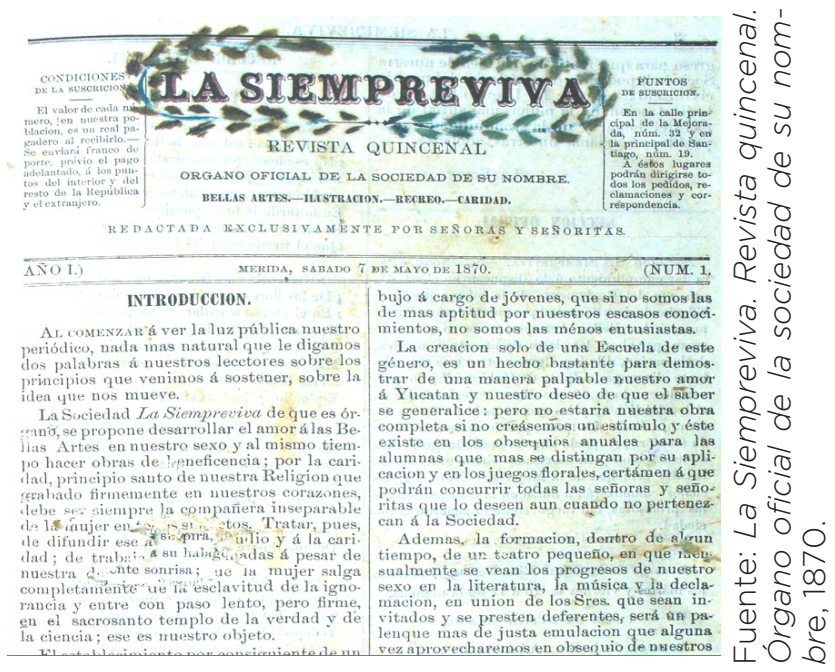


Esta demanda, ya presente desde los años 40 en las primeras publicaciones femeninas, tuvo uno de sus mayores momentos de algidez precisamente durante la década de 1880, en la voz de la guerrerense Laureana Wright González (1910), quien además de dirigir una revista que mencionaremos más adelante, Violetas del Anáhuac, dedicó a dicha discusión un vasto conjunto de artículos que a partir de 1880, y hasta 1907, se publicaron en diversos semanarios, entre ellos La llustración femenil.

Como es sabido, no fue ella la única escritora mexicana que dedicó a esta temática muchas horas, tinta y papel. Otras importantes autoras del periodo, como Dolores Correa Zapata, la ya mencionada Rita Cetina y Laura Méndez de Cuenca produjeron también muchos textos al respecto. Creo, sin embargo, que la escritura de Laureana nos transmite con vehemencia el sentido vitalmente transformador de esa coyuntura, de ese momento histórico en el que todas ellas participaron como protagonistas directas. Me refiero a la conformación de un nuevo sentido de pertenencia cultural de las mexicanas: el de la escritora y de sus implicaciones en su emergencia como protagonistas de la vida pública.

En este sentido, la trascendencia del discurso de Laureana radica, entre otros aspectos, en haber logrado evadir la lógica hasta entonces común utilizada para justificar la desigualdad social entre los sexos (la diferencia anatómica) para, situándose en un horizonte estrictamente sociocultural, señalar un sentido de pertenencia genérica vinculado con la construcción de una identidad femenina desde su lugar como un sujeto cultural y, por lo tanto, no predestinado por sus presuntas capacidades o limitaciones biológicas.

En otras palabras, Laureana Wright fue más allá de la idea entonces imperante sobre la diferencia sexual (léase biológica, anatómica o corporal) como el eje a partir del cual se determinaban las conductas, actividades y espacios sociales propios de cada sexo. Por el contrario, afirmó que la única diferencia sustancial entre hombres y mujeres era la desigualdad con respecto al nivel de instrucción recibida (es decir, de los conocimientos formales adquiridos en la escuela). Una desigualdad que, en efecto, se traducía en un desarrollo desigual de las habilidades intelectuales y cognitivas entre un sexo y otro. Asimismo, insistió en que la verdadera transformación de dicho escenario radicaba no sólo en la modificación de las estructuras legales y materiales que restringían el acceso de las mujeres a una educación superior, sino todavía más: en la reformulación misma de la identidad femenina subyacente en la conciencia, en la subjetividad de cada mujer.

Desde dicho horizonte de reflexión, la autora identificó la imprescindible necesidad de gestar una confianza interna que permitiera transformar de raíz la introyección de la idea de la desigualdad entre los sexos, un proceso de autoafirmación sin el cual no sería posible que las mujeres transitaran hacia un modelo de educación que promoviera el desarrollo de sus habilidades intelectuales. El horizonte subjetivo de dicha transformación se explicita con puntualidad en este breve fragmento, que pertenece a uno de los muchos apartados que componen Educación errónea de la mujer, uno de sus textos más importantes:

Proclamar dentro de los límites del decoro y la justicia la emancipación de la mujer, la igualdad de la educación, de principios y de derechos entre ambos sexos por medio de una revolución intelectual que la mujer tiene que efectuar por sí misma y con el auxilio de sus propias fuerzas, como se efectúan todas las revoluciones [pretende] hacerla apta para atenderse y bastarse a sí misma, dándole la instrucción por salvaguardia, el trabajo por recurso y la dignidad por égida [...] ¿Qué necesita la mujer para llegar a esta perfección? Fuerza de voluntad, valor moral, amor a la instrucción y, sobre todo, amor a símisma y a su sexo, para trabajar por él, para rescatarle de los últimos restos de esclavitud que por inercia conserva (Alvarado, 2005: 95, 120).

Esta misma convicción atraviesa las páginas de todas las publicaciones que dieron 
voz a las mujeres a lo largo de las tres últimas décadas del siglo XIX y las primeras del $X X$, de manera especial en las 12 dirigidas por mujeres (impresas en su mayoría en la Ciudad de México) y cuyas directoras, cabe recordar, tenían ya entonces más de 20 años dentro del mercado de los medios impresos. Doce publicaciones representativas de esta etapa, y que mencionamos brevemente, son: la ya referida La Siempreviva (1870-1872); las Hijas del Anáhuac (18731874), administrada y dirigida literariamente por Concepción García y Ontiveros (autonombrada "redactora en jefe") y elaborada en las prensas de la Escuela de Artes y Oficios para Mujeres; El Recreo del Hogar (1879), publicada en Tabasco por las manos de las ya también mencionadas Rita Cetina y Gertrudis Tenorio.

Para la década de los 80 tenemos El Á/bum de la mujer (1883-1890), proyecto editorial comandado durante siete años por la española avecindada en México Concepción Gimeno de Flacquer.

\section{Figura 2 Portada de El Álbum de la Mujer}

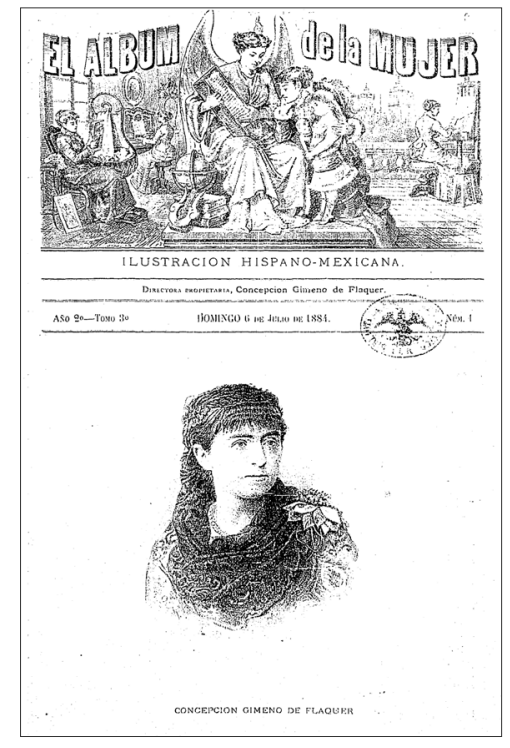

Fuente: El Álbum de la Mujer (1884).

La República Literaria (1886-1890) era codirigida por Esther Tapia de Castellanos -autora del primer libro de poesía femenina publicado en nuestro país, Flores Silvestres, de 1871- y José López Portillo y Rojas; la ya mencionada Violetas del Anáhuac, que bajo la sucesiva dirección de Laureana Wright y Mateana Murguía de Aveleyra se publicó en la Ciudad de México durante dos años (1887-1889).

Figura 3 Portada de Las Hijas del Anáhuac

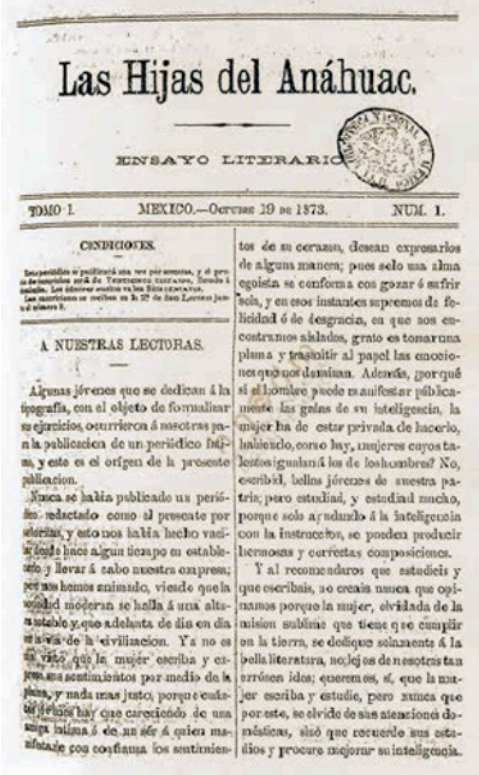

Fuente: Las Hijas del Anáhuac (1873).

Figura 4 Portada de Violetas del Anáhuac

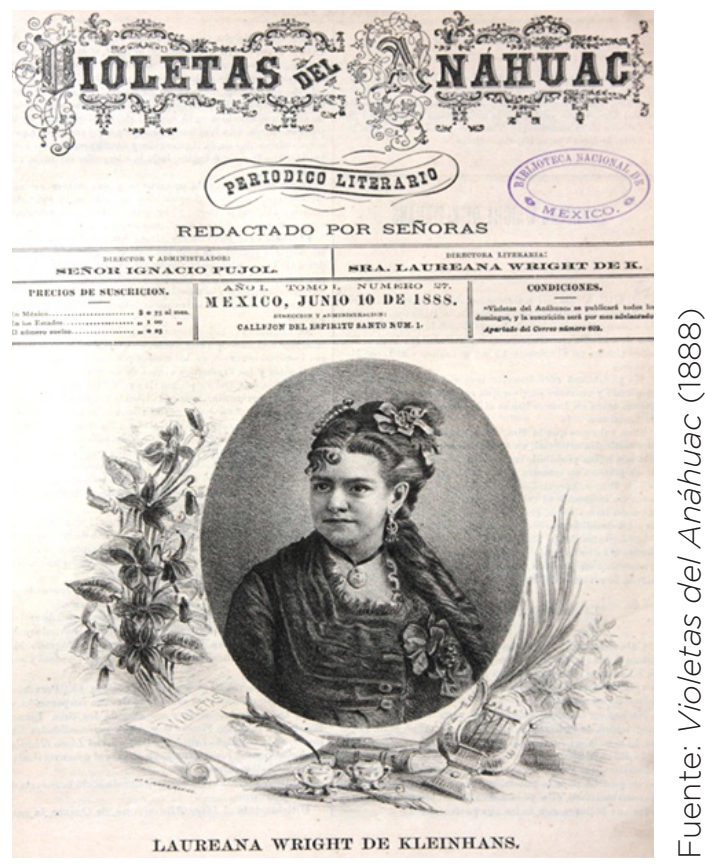


La Palmera del Valle (1887), de Guadalajara, Jalisco, dirigida por la profesora Refugio Barragán de Toscano, la primera novelista mexicana -La hija del bandido o Los subterráneos del Nevado, publicada en 1886- y autora del segundo libro de poesía femenina publicado en suelo nacional (Celajes de Occidente, de 1880).

\section{Figura 5}

\section{Portada de La Palmera del Valle}

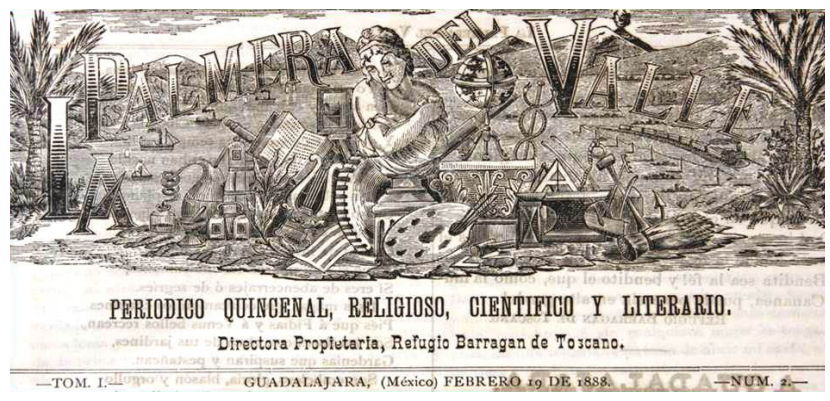

Fuente: La Palmera del Valle (1888).

Son las citadinas Guadalupe Fuentes viuda de Gómez Vergara e Isabel M. viuda de Gamboa quienes, ya en las postrimerías de los años noventa, 1896, publican El Periódico de las Señoras, primer semanario femenino que, desde una preclara mirada empresarial, se propone convertir este proyecto editorial en una actividad rentable.

\section{Figura 6 Portada de El periódico de las Señoras}

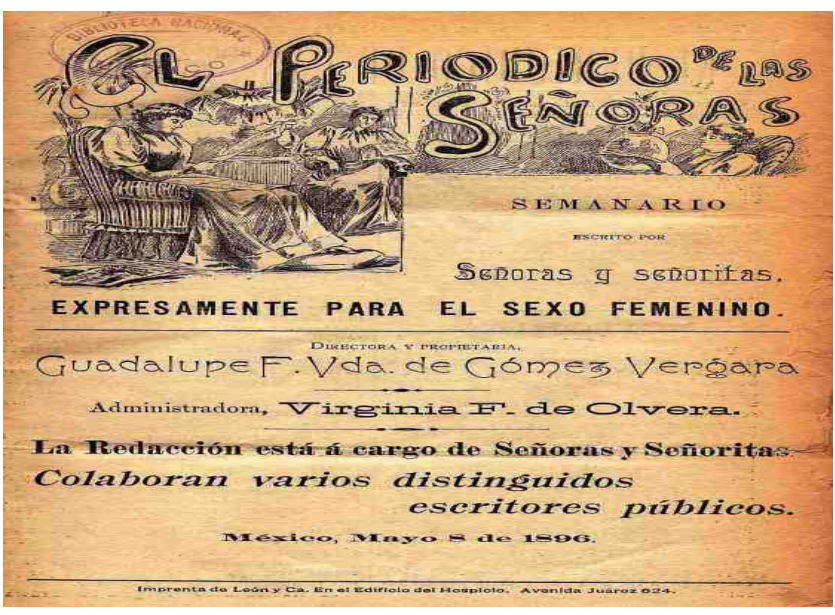

Fuente: El periódico de las Señoras (1896).
La publicación que cierra el proceso atendido a lo largo de estas páginas es La Mujer Mexicana (1904-1907), revista mensual dirigida sucesivamente por Victoria Sandoval de Zarco, Luz Fernández viuda de Herrera y las ya varias veces referidas Dolores Correa Zapata y Laura Méndez de Cuenca; resume con elocuencia el camino trazado por todas las publicaciones que en su género le anteceden, y claro, por la pluma y el esfuerzo de las escritoras que les dieron vida.

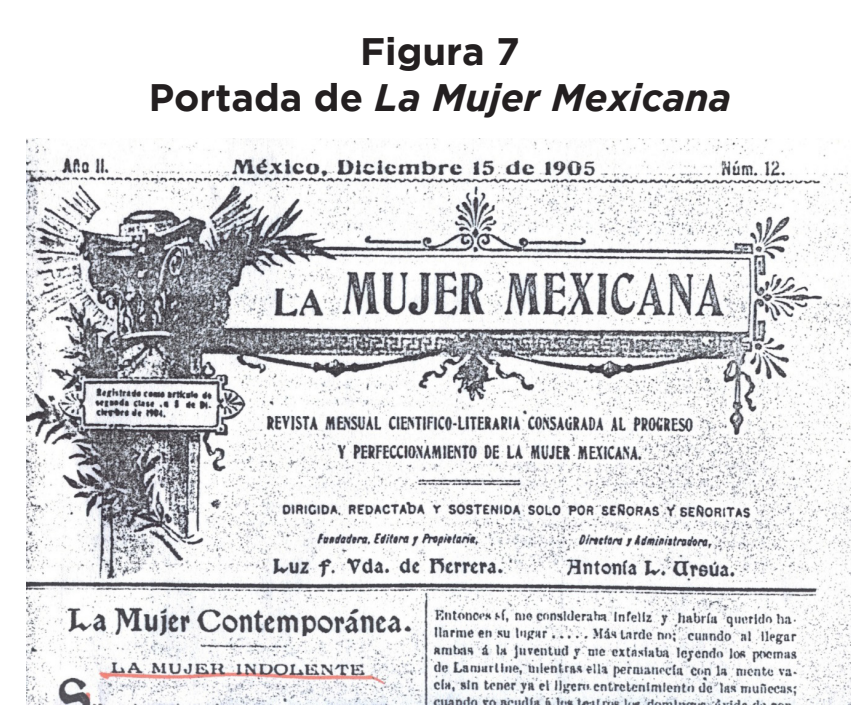

Fuente: La Mujer Mexicana (1905).

Ello es así porque, en primer término, sus páginas expresan con claridad el planteamiento ya citado en la voz de Laureana Wright, sobre la inteligencia femenina y el derecho de las mujeres a desplegarla mediante su ingreso a la educación superior. Asimismo, porque alrededor suyo se agrupa una buena parte de las fundadoras (y colaboradoras) de la última generación de revistas citadas y escritoras más jóvenes que durante las primeras décadas del siglo $\mathrm{XX}$ emprenden proyectos semejantes, o que ya dedicadas del todo al oficio de la escritura, colaboran en múltiples revistas e incursionan en diversos géneros de la narrativa literaria. Entre otras, Trinidad Orcilles, Matilde Montoya, Dolores Jiménez, Antonia L. Ursúa, Severa Aróstegui, María Enriqueta Camarillo y de nueva cuenta Dolores Correa Zapata, Laura Méndez de Cuenca y Mateana Murguía, quienes para dicho momento con- 
taban ya con el reconocimiento de diversos círculos literarios.

En segundo lugar, porque muchos de los textos publicados (ya desde la poesía, el ensayo o la crónica) abordan desde diversos ángulos la demanda del reconocimiento de las mujeres como ciudadanas. Así, por ejemplo, las páginas de esta revista dan cuenta de uno de los primeros actos colectivos en pro de ese horizonte, la creación de la Sociedad Protectora de la Mujer, primera asociación de mujeres en México que se autodenominó feminista. Fundada en la Ciudad de México en 1904, y conformada en su mayoría por las colaboradoras de La Mujer Mexicana, esta agrupación promovió su demanda de "emancipación racional y justa" no sólo a través de la escritura, sino también mediante la creación de círculos de estudio, un consultorio médico gratuito, y de manera muy especial, la fundación de la Sociedad Protectora de la Mujer.

A semejanza de la escuela impulsada por las Siemprevivas meridanas, esta agrupación formó parte de un proyecto sociocultural con amplias expectativas. Anunciada como una "naciente sociedad feminista", esta asociación refrendó la búsqueda de una "confraternidad femenina" que, entre muchos otros objetivos, estableciera "una emancipación racional y justa”. Así lo expresó Laura S. de Bolaños Torres en el discurso inaugural de esta singular asociación:

Señoras y Señoritas: me es altamente honroso tener la satisfacción de estar entre vosotras esta mañana y dirigiros la palabra, aunque conozco mis inaptitudes. Brilla en vuestras frentes la luminosa antorcha del saber y creo un deber mío, rendiros el homenaje que merecéis por vuestro talento, vuestra ilustración y vuestros arduos trabajos en la brega bendita que habéis emprendido, luchando denonadas, anhelosas y perseverantes, por ocupar los altos puestos que tan justa y legítimamente desempeñáis en la presente etapa de adelanto y civilidad. Es tan noble y santo el asunto que nos ha reunido aquí [...] ideal que hace mucho tiempo acaricio en mi alma, la idea de unir las fuerzas intelectuales de las mujeres mexicanas y hacer surgir en nuestra patria, en la evolución asombrosa del presente siglo, la tea encendida de la confraternidad femenina [...] Que la unión sea la que nos dé valor para lanzarnos a santas empresas, a los trabajos atrevidos del intelecto o a las elucubraciones sublimes del arte [...] Necesitamos asociarnos, formar una colectividad en donde las mujeres hallemos enseñanzas y nos apoyemos mutuamente [formemos] una sociedad que ampare y defienda los derechos de la mujer en México, que la haga más respetable y respetada, al mismo tiempo que le dé ocasión para que su talento se revele. Constituid esa colectividad [...] Llegará un día en que nos congratulemos por haber sido las primeras en ponernos a la altura que demanda de nosotras el actual estado de cosas, y el avance general de los pueblos (Bolaños Torres de, 1904.)

En tercer lugar, la actividad que desde el magisterio desarrollaron muchas de estas primeras escritoras y fundadoras de las primeras revistas para mujeres da cuenta, una vez más, de la inseparable relación entre el proceso de apropiación de la escritura y la demanda de acceso a la educación. En más de un sentido, no es fortuito que la mayor parte de los proyectos editoriales ya mencionados fueran gestados, o apoyados de manera directa, por quienes también fueron pioneras en acreditarse como profesoras de primeras letras (único nivel de formación magisterial al que inicialmente tuvieron acceso las mujeres en México), y cuyos nombres hemos ya referido: Rita Cetina, Dolores Correa Zapata, Mateana Murguía de Aveleyra, Refugio Barragán de Toscano, Esther Tapia de Castellanos, Concepción García y Ontiveros, Josefina Pérez de García Torres, Laura Méndez de Cuenca, María Enriqueta Camarillo.

\section{Reflexión final}

La lectura es el engrandecimiento (del alma); su noble horizonte, su perfección. Ella hace que nos remontemos a los altos espacios de la imaginación; que atravesemos, cruzando por entre variados panoramas, los siglos remotos 
de lo pasado; que nos adelantemos al tiempo, y partiendo de las probables deducciones de lo presente, penetremos en los velados siglos de lo porvenir. La lectura cultiva el cerebro y el corazón a la par: enseña y moraliza, instruye y recrea. Los libros son el tiempo, el espacio, el infinito, a donde a cualquiera hora podemos penetrar. Ellos son, en fin, el vasto kaleidoscopio, el encantado cosmorama que hace eterna la idea e inmortal el pensamiento que la creó [lo impreso] es el reinado indestructible del pensamiento y la sabiduría (Wright, 1888: 294).

Hace ya más de un siglo, en septiembre de 1910, como parte de las celebraciones realizadas para conmemorar el primer centenario de la independencia nacional, la Secretaría de Instrucción Pública y Bellas Artes auspició la publicación de Mujeres Notables Mexicanas (Wright, 1910), obra que a lo largo de 534 páginas nos legó un testimonio sobre la vida de 120 mujeres que, a partir de ese momento, al menos en términos simbólicos, se adentraron formalmente en la memoria histórica de nuestro país.

Este libro, en su género el primero, fue uno de los textos póstumos de Laureana Wright González, más de una vez referida a lo largo de estas páginas. Mejor conocida por su apellido de casada, Wright de Kleinhans, no vivió lo suficiente para contemplar este conjunto de historias que, al menos durante los últimos 16 años de su vida, había comenzado a publicar en distintas revistas literarias y, de manera particular, en Violetas del Anáhuac.

La publicación de Mujeres Notables Mexicanas, un tributo a la memoria de esta mujer, cuyo declarado amor por las letras la llevó a incursionar lo mismo en la prosa y la poesía, que en el ensayo histórico, filosófico, científico y moral, fue también una expresión palpable del victorioso ingreso en el imaginario cultural de México, de aquella identidad por la que tanto habían luchado ella y sus contemporáneas, y más atrás, sus madres, sus abuelas y las abuelas de sus tatarabuelas: la mujer escritora.

Una identidad de lo femenino que concebía a las mujeres como sujetos históricos, como individuos con un papel activo en la construcción social y cultural del país al que pertenecían. En otras palabras, por fin, en ese libro, quedaba registrada por escrito -e impresa- la memoria de aquel conjunto de mujeres que, más allá del origen étnico, la edad, la condición social, la adscripción ideológica, la creencia espiritual o la actividad desarrollada en vida, merecían ser adjetivadas como notables, merecían ser recordadas por las generaciones futuras. ¿Por qué eran dignas de tal atención? Porque habían dado cuenta de su capacidad de hablar con una propia voz, de actuar y decidir por sí y para sí mismas.

Tal es, entre muchas otras, la trascendencia cultural de este libro. Cada una de sus páginas nos recuerda la batalla librada por aquellas mujeres, y la conquista final del territorio fértil sobre el cual es posible que muchas mexicanas hoy construyan su vida: la creación de una identidad femenina que se reconoce a sí misma como un sujeto cultural, como un individuo que participa activamente en la generación y el registro diario de la historia, de la suya, la de todos los días, y también, la de la historia de su país.

Para recordarlo, bien vale la pena adentrarse en sus páginas y, además de conocer la historia de las mujeres que habitan en ellas, reconocer que gracias a las transformaciones gestadas por esta primera conquista, el acceso de las mexicanas a todos los niveles y ámbitos de la educación es hoy un derecho constitucional, y que la escritura permanece como una de las herramientas más poderosas para gestar el cambio y la transformación.

Como consigna Piedad Peniche (2015), el profesor Rodolfo Menéndez, primer biógrafo de Rita Cetina, quien además del proyecto escolar de La Siempreviva, dirigió durante 18 años el Instituto Literario de niñas (primera escuela pública para niñas en Mérida), afirmó que muchas de las mujeres que participaron en el Primer Congreso Feminista que tuvo lugar en Mérida, Yucatán, el 13 de enero de 1916 (en su mayoría profesoras) "habían pasado por las aulas de doña Rita Cetina Gutiérrez" (Peniche, 2015: 61). 
Cierto o no, es indudable que al igual que todas las acciones realizadas por ella y todas las mujeres rememoradas a lo largo de estas páginas, sentaron un precedente fundamental en la transformación del panorama educativo para las mexicanas.

En ese sentido, definitivamente no es una casualidad que años después, en 1919, y como parte de los aires de cambio impulsados por estas precursoras, a iniciativa de Elvia Carrillo Puerto, la primera liga feminista y sufragista en México recibiera el nombre de "Rita Cetina", para honrar así la memoria de esta ilustre maestra y escritora, cuyas acciones y palabras escritas formaron parte de la dura labranza que abrió el camino a los movimientos organizados de mujeres y el feminismo en nuestro país.

\section{Fuentes consultadas}

Alamán, Lucas (1831), "Sin título", Registro Oficial del Gobierno de los Estados Unidos Mexicanos, 14 de marzo, Ciudad de México.

Alvarado, Lourdes (2005), "Transcripción y estudio introductorio", en Educación y superación femenina en el siglo XIX: dos ensayos de Laureana Wright, Ciudad de México, UNAM, (Cuadernos del Archivo Histórico de la UNAM, 19).

Bolaños Torres de, Laura S. (1904), "Realidades. Alocución pronunciada en la primera sesión de la naciente Sociedad Feminista", La Mujer Mexicana, tomo I, núm. 3, 1 de marzo, Ciudad de México.

Infante Vargas, Lucrecia (2017), De la escritura al margen a la dirección de empresas culturales: mujeres en la prensa literaria mexicana del siglo XIX (18051907), Sevilla, Ediciones Benilde.

Infante Vargas, Lucrecia (2014), "Las mujeres y el arribo del liberalismo en México: antecedentes, cambios, permanencias e impactos (1753-1850)", en Adriana Maza (coord.), De liberales a liberadas. Pensamiento y movilización de las mujeres en la historia de México
(1753-1975), Ciudad de México, Nueva Alianza.

Muriel, Josefina (2000), Cultura femenina novohispana, Ciudad de México, UNAM-Instituto de Investigaciones Históricas.

Muriel, Josefina (1946), Conventos de monjas en la Nueva España, Ciudad de México, Ed. Santiago.

Peniche, Piedad (2015), Rita Cetina. La Siempreviva y el Instituto Literario de Niñas: una cuna del feminismo mexicano, 1846-1908, Ciudad de México, INEHRM.

"Pepita" (1810), "Carta de una señorita a otra, que se halla ausente de esta ciudad", Diario de México, 3 de septiembre, Ciudad de México.

Vicario, Leona (1831), "Carta de Leona Vicario dirigida a Lucas Alamán", El Federalista Mexicano, 2 de abril, Ciudad de México, Imprenta de Sabino Ortega.

Wright, Laureana (1910), Mujeres Notables Mexicanas, Ciudad de México, Secretaría de Instrucción Pública y Bellas Artes.

Wright, Laureana (1888), "Los libros", Violetas del Anáhuac, 27 de mayo, Ciudad de México.

\section{Hemerografía}

Diario de México, Ciudad de México, 18051815.

El Álbum de la Mujer, Ciudad de México, 1883-1890.

El Federalista Mexicano, Ciudad de México, 1831.

El Periódico de las Señoras, Ciudad de México, 1896.

La Mujer Mexicana, Ciudad de México, 19041907. 
La Palmera del Valle, Guadalajara, Jalisco, 1887.

La Siempreviva, Mérida, Yucatán, 1870-1872.

Las Hijas del Anáhuac, Ciudad de México, 1887.

Registro Oficial del Gobierno de los Estados Unidos Mexicanos, Ciudad de México, 1831.

Violetas del Anáhuac, Ciudad de México, 1887-1889.

Recibido: 7 de noviembre de 2021. Aceptado: 14 de diciembre de 2021.

Publicado: 13 de enero de 2022.

\section{Lucrecia Infante Vargas}

Es doctora en Historia por la Universidad Nacional Autónoma de México (UNAM). Actualmente es profesora investigadora en la Facultad de Filosofía y Letras de la UNAM. Sus líneas de investigación son: Historia cultural y política de las mujeres en México, siglos XIX y XX; Mujeres y cultura impresa, siglos XIX y XX, y Vínculos teórico-metodológicos de la Historia y la Literatura. Entre sus más recientes publicaciones destacan, como autora: "José María Vigil: promotor, crítico e historiador de la literatura femenina decimonónica", en Miguel Ángel Castro (coord.) José María Vigil. A cien años de su muerte, Ciudad de México, UNAM-IIB, pp. 193-203 (2018); De la escritura al margen a la dirección de empresas culturales: mujeres en la prensa literaria mexicana del siglo XIX (1805-1907), Sevilla, Benilde Ediciones (2017); como coautora: Del Kindergarten al jardín de niños. Un proyecto de mujeres en la historia de la educación de México (19041928), Ciudad de México, SNTE (2016). 